\title{
M-curves of degree 9 with deep nests
}

\author{
Séverine Fiedler-Le Touzé
}

September 22, 2018

\begin{abstract}
The first part of Hilbert's sixteenth problem deals with the classification of the isotopy types realizable by real plane algebraic curves of given degree $m$. For $m \geq 8$, one restricts the study to the case of the $M$-curves. For $m=9$, the classification is still wide open. We say that an $M$-curve of degree 9 has a deep nest if it has a nest of depth 3 . In the present paper, we prohibit 10 isotopy types with deep nest and no outer ovals.
\end{abstract}

\section{Introduction}

Let $A$ be a real algebraic non-singular plane curve of degree $m$. Its complex part $\mathbb{C} A \subset \mathbb{C} P^{2}$ is a Riemannian surface of genus $g=(m-1)(m-2) / 2$; its real part $\mathbb{R} A \subset \mathbb{R} P^{2}$ is a collection of $L \leq g+1$ circles embedded in $\mathbb{R} P^{2}$. If $L=g+1$, we say that $\mathrm{A}$ is an M-curve.A circle embedded in $\mathbb{R} P^{2}$ is called oval or pseudo-line depending on whether it realizes the class 0 or 1 of $H_{1}\left(\mathbb{R} P^{2}\right)$.If $m$ is even, the $L$ components of $\mathbb{R} A$ are ovals; if $m$ is odd, $\mathbb{R} A$ contains exactly one pseudo-line, which will be denoted by $\mathcal{J}$. An oval separates $\mathbb{R} P^{2}$ into a Möbius band and a disc. The latter is called the interior of the oval. An oval of $\mathbb{R} A$ is empty if its interior contains no other oval. One calls outer oval an oval that is surrounded by no other oval. Two ovals form an injective pair if one of them lies in the interior of the other one. We call nest of depth $d+1$ a configuration of ovals $\left(O_{0}, O_{1}, \ldots, O_{d}\right)$ such that $O_{i}$ lies in the interior of $O_{j}$ for all pairs $i, j$, with $j>i$. An $M$-curve of degree $m=2 k$ or $m=2 k+1$ is said to have a deep nest if it has a nest of depth $k-1$.Notice that for a curve with deep nest, all of the other ovals are empty by Bezout's theorem with an auxiliary line.

Let us call the isotopy type of $\mathbb{R} A \subset \mathbb{R} P^{2}$ the real scheme of $A$; it will be described with the following notation due to Viro. The symbol $\langle\mathcal{J}\rangle$ stands for a curve consisting in one single pseudo-line; $\langle n\rangle$ stands for a 
curve consisting in $n$ empty ovals. If $X$ is the symbol for a curve without pseudo-line, $1\langle X\rangle$ is the curve obtained by adding a new oval, containing all of the others in its interior. Finally, a curve which is the union of 2 disjoint curves $\langle A\rangle$ and $\langle B\rangle$, having the property that none of the ovals of one curve is contained in an oval of the other curve, is denoted by $\langle A \amalg B\rangle$. The classification of the real schemes which are realizable by $M$-curves of a given degree in $\mathbb{R} P^{2}$ is part of Hilbert's sixteenth problem. This classification is complete up to degree 7 and almost complete in degree 8. A systematic study of the case $m=9$ has been done, the main contribution being due to A. Korchagin. See e.g. [5], 7], 8, , 9], [11] for the constructions, and [5, 6], [1], 3], 12], [13] for the restrictions. The main result of the present paper is the prohibition of 10 new schemes. The proof is an improvement of the classical restriction method: we use Bezout's theorem with auxiliary pencils of rational cubics, and add to the classical theorems on complex orientations the newer formulas of Orevkov [10] for $M$-curves with deep nests.

Let us briefly recall some facts about complex orientations. The complex conjugation conj of $\mathbb{C} P^{2}$ acts on $\mathbb{C} A$ with $\mathbb{R} A$ as fixed points sets. Thus, $\mathbb{C} A \backslash$ $\mathbb{R} A$ is connected, or splits in 2 homeomorphic halves which are exchanged by conj. In the latter case, we say that $A$ is dividing. Let us now consider a dividing curve $A$ of degree $m$, and assume that $\mathbb{C} A$ is oriented canonically. We choose a half $\mathbb{C} A_{+}$of $\mathbb{C} A \backslash \mathbb{R} A$. The orientation of $\mathbb{C} A_{+}$induces an orientation on its boundary $\mathbb{R} A$. This orientation, which is defined up to complete reversion, is called complex orientation of $A$. One can provide all the injective pairs of $\mathbb{R} A$ with a sign as follows: such a pair is positive if and only if the orientations of its 2 ovals induce an orientation of the annulus that they bound in $\mathbb{R} P^{2}$. Let $\Pi_{+}$and $\Pi_{-}$be the numbers of positive and negative injective pairs of $A$. If $A$ has odd degree, each oval of $\mathbb{R} A$ can be provided with a sign: given an oval $O$ of $\mathbb{R} A$, consider the Möbius band $\mathcal{M}$ obtained by cutting away the interior of $O$ from $\mathbb{R} P^{2}$. The classes $[O]$ and $[2 \mathcal{J}]$ of $H_{1}(\mathcal{M})$ either coincide or are opposite. In the first case, we say that $O$ is negative; otherwise $O$ is positive. Let $\Lambda_{+}$and $\Lambda_{-}$be respectively the numbers of positive and negative ovals of $\mathbb{R} A$. The complex scheme of $A$ is obtained by enriching the real scheme with the complex orientation: let e.g. $A$ have real scheme $\langle\mathcal{J} \amalg 1\langle\alpha\rangle \amalg \beta\rangle$. The complex scheme of $A$ is encoded by $\left\langle\mathcal{J} \amalg 1_{\epsilon}\left\langle\alpha_{+} \amalg \alpha_{-}\right\rangle \amalg \beta_{+} \amalg \beta_{-}\right\rangle$where $\epsilon \in\{+,-\}$ is the sign of the non-empty oval; $\alpha_{+}, \alpha_{-}$are the numbers of positive and negative ovals among the $\alpha ; \beta_{+}, \beta_{-}$are the numbers of positive and negative ovals among the $\beta$ (remember that all signs are defined with respect to the orientation of the pseudo-line $\mathcal{J}$ ). 
Rokhlin-Mishachev formula: If $m=2 k+1$, then

$$
2\left(\Pi_{+}-\Pi_{-}\right)+\left(\Lambda_{+}-\Lambda_{-}\right)=L-1-k(k+1)
$$

Fiedler theorem: Let $\mathcal{L}_{t}=\left\{L_{t}, t \in[0,1]\right\}$ be a pencil of real lines based in a point $P$ of $\mathbb{R} P^{2}$. Consider two lines $L_{t_{1}}$ and $L_{t_{2}}$ of $\mathcal{L}_{t}$, which are tangent to $\mathbb{R} A$ in two points $P_{1}$ and $P_{2}$, such that $P_{1}$ and $P_{2}$ are related by a pair of conjugated imaginary arcs in $\mathbb{C} A \cap\left(\bigcup L_{t}\right)$.

Orient $L_{t_{1}}$ coherently to $\mathbb{R} A$ in $P_{1}$, and transport this orientation through $\mathcal{L}_{t}$ to $L_{t_{2}}$. Then this orientation of $L_{t_{2}}$ is compatible to that of $\mathbb{R} A$ in $P_{2}$.

Orevkov formulas for odd degree Let $A$ be an $M$-curve of degree $m=$ $2 k+1$, with a deep nest. Let $l_{+}$and $l_{-}$be respectively the numbers of positive and negative non-empty ovals; $\lambda_{+}$and $\lambda_{-}$be respectively the numbers of positive and negative empty ovals. Let $\pi_{s}^{S}, S, s \in\{+,-\}$ be the number of pairs $(O, o)$ where $o$ is an empty oval surrounded by $O$ and $(S, s)$ are the signs of $(O, o)$. Then:

$\pi_{-}^{+}-\pi_{+}^{+}=\left(l_{+}\right)^{2}$, and $\pi_{+}^{-}-\pi_{-}^{-}+\left(\lambda_{+}-\lambda_{-}\right) / 2=\left(l_{-}\right)^{2}+l_{-}$

\section{Restrictions}

\subsection{Definitions and results}

Let $C_{9}$ be an $M$-curve of degree 9 .Given an empty oval $X$ of $C_{9}$, we often will have to consider one point chosen in the interior of $X$. For simplicity, we shall call this point also $X$. We denote the pencil of lines based in $X$ by $\mathcal{F}_{X}$. Let $X, Y$ be 2 empty ovals of $C_{9}$. We shall denote by $[X Y]$ (resp. $[X Y]^{\prime}$ ) the segment of line $X Y$ that cuts $\mathcal{J}$ an even (resp. an odd) number of times. We say that $[X Y]$ is the principal segment determined by $X, Y$. Let $X, Y, Z$ be three empty ovals of $C_{9}$. Corresponding three points $X, Y$ and $Z$ determine 4 triangles of $\mathbb{R} P^{2}$. We will call principal triangle and denote by $X Y Z$ the triangle bounded by the segments $[X Y],[Y Z]$ and $[X Z]$. We denote respectively by $\mathcal{F}_{X}: Y \rightarrow Z$ and $\mathcal{F}_{X}^{\prime}: Y \rightarrow Z$ the pencils of lines based in $X$ that sweep out the segments $[Y Z]$ and $[Y Z]^{\prime}$. We denote by $\mathcal{F}_{X}^{\epsilon}: Y \rightarrow Y \epsilon \in\{ \pm\}$ the complete pencils rotating in either direction, $\epsilon=+$ being chosen for the counter-clockwise direction. If $Y_{1}, \ldots, Y_{n}$ are empty ovals met successively by $\mathcal{F}_{X}^{\epsilon}: Y_{1} \rightarrow Y_{1}$, we say that this pencil has one $\mathcal{J}$-jump between 2 consecutive ovals, say $Y_{i}, Y_{i+1}$ if $\mathcal{F}_{X}^{\epsilon}: Y_{i} \rightarrow Y_{i+}$ sweeps out the segment $\left[Y_{i} Y_{i+1}\right]^{\prime}$. Notice that the total number of $\mathcal{J}$-jumps 


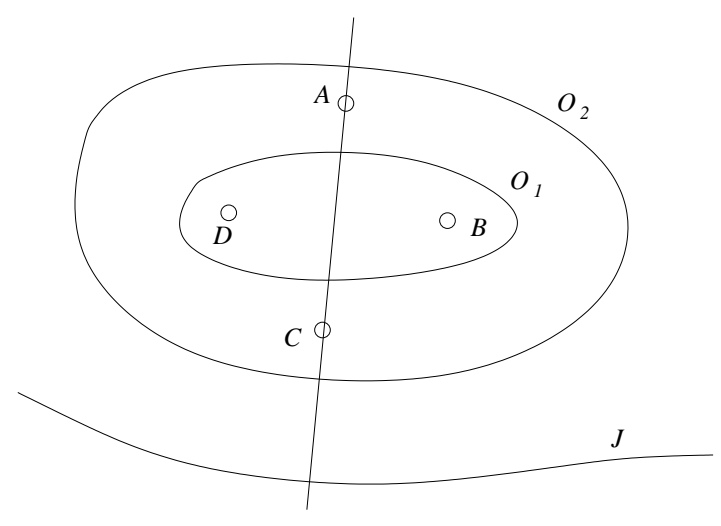

Figure 1: $O_{1}$-jump

of a complete pencil over a sequence of ovals is always odd and does not depend on the choices of $\epsilon$ and $Y_{1}$. Denote by $\left(L_{t}\right), t \in[0,1]$ the lines of the pencil $\mathcal{F}_{X}^{\epsilon}: Y_{1} \rightarrow Y_{n}$. We say that $Y_{1}, \ldots, Y_{n}$ form a Fiedler chain with respect to this pencil if each $Y_{i}, i=1, \ldots, n-1$ is connected to $Y_{i+1}$ by a pair of imaginary arcs of $\mathbb{C} A \cap\left(\bigcup L_{t}\right)$. Notice that by Fiedler's theorem, the signs of $Y_{i}$ and $Y_{i+1}$ coincide if and only if the pencil $\mathcal{F}_{X}^{\epsilon}: Y_{i} \rightarrow Y_{i+1}$ has a $\mathcal{J}$-jump.

An ordered group of empty ovals $A_{1}, \ldots, A_{n}$ of $C_{9}$ lies in a convex position if for each triple $A_{i}, A_{j}, A_{k}$, the principal triangle $A_{i} A_{j} A_{k}$ does not contain any other oval of the group and $A_{1}, \ldots, A_{n}$ are the successive vertices of $\bigcup A_{i} A_{j} A_{k}$ (the convex hull of the group).

Let $C_{9}$ be an $M$-curve with real scheme $\langle\mathcal{J} \amalg \alpha \amalg 1\langle\beta \amalg 1\langle\gamma\rangle\rangle\rangle$. We shall call the ovals of the groups $\alpha, \beta$ and $\gamma$ respectively outer, median and inner ovals. Notice that the inner ovals must lie in convex position. Otherwise, let $A, B, C, D$ be 4 inner ovals such that $D$ lies inside of the principal triangle $A B C$.Then, for any choice of a fifth empty oval $E$, the conic through $A, B, C, D, E$ cuts $C_{9}$ in more than 18 points. This is a contradiction.

Definition 1 We say that $C_{9}$ has a $O_{1}$-jump if there exist 2 median ovals $A, C$ and 2 inner ovals $B, D$, such that the line $A C$ separates $B$ from $D$ in $\operatorname{Int}\left(O_{1}\right)$.

Notice, again with auxiliary conics, that if $C_{9}$ has a $O_{1}$-jump determined by $A, B, C, D$, then the principal segment $[A C]$ cuts $O_{1}$, as shown in Figure 1. The lines $(A B),(B C),(C D),(D A)$ give rise to 3 quadrangles and 4 triangles. All of the remaining empty ovals lie in the union of the 4 triangles. 


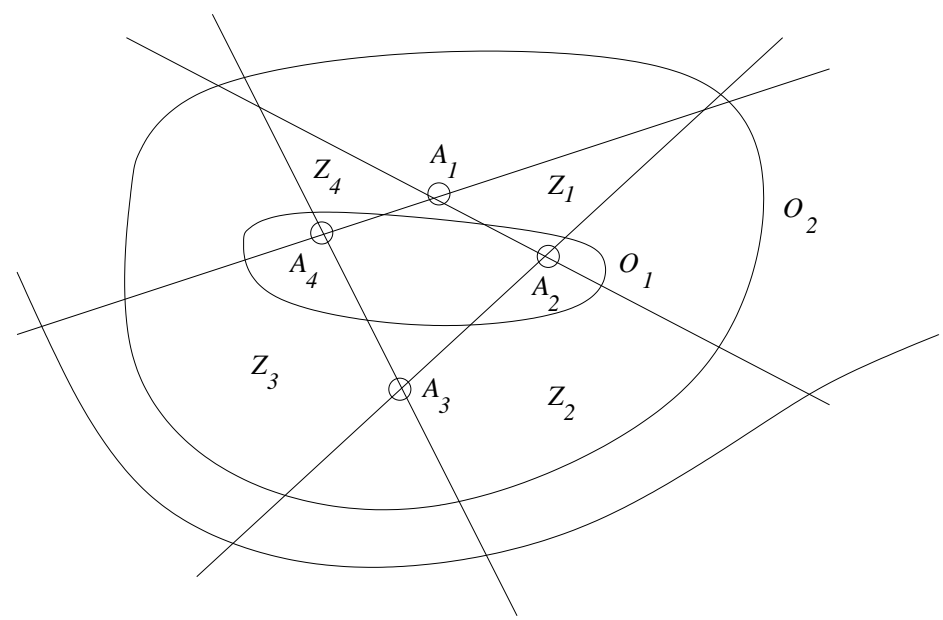

Figure 2: $C_{9}$ with $J=1$

Lemma 1 If $C_{9}$ has a median oval $A_{1}$ such that the pencil $\mathcal{F}_{A_{1}}$ sweeping out $O_{1}$ meets successively empty ovals $A_{2}, A_{3}, \ldots, A_{2 J+2}$, where the $A_{i}, i$ even (resp. odd) are inner (resp. median) ovals. Then:

(1) The $A_{i} i=1, \ldots, 2 J+2$ lie in convex position.

(2) Let $A_{i}, A_{j}, A_{k}, A_{l}$ be 4 consecutive ovals in the cyclic ordering, we denote by $T_{j}$ the triangle determined by the lines $\left(A_{i} A_{j}\right)\left(A_{j} A_{k}\right)\left(A_{k} A_{l}\right)$ with edge $\left[A_{j} A_{k}\right]$, that does not intersect the convex hull of $A_{1}, A_{2}, \ldots, A_{2 J+2}$. All of the remaining empty ovals lie in $\bigcup T_{j}, j=1, \ldots, 2 J+2$; if an oval $A$ lies in $T_{j} \cap \operatorname{Int}\left(\mathrm{O}_{2}\right), A$ is not separated in $T_{j}$ from the edge $\left[A_{j} A_{k}\right]$ by $\mathcal{J}$.

(3) There is a natural cyclic ordering of the empty ovals. This ordering is given by: the complete pencils of lines $\mathcal{F}_{B}$, for any inner oval $B$, and the pencils $\mathcal{F}_{A}$, where $A$ is a median oval in some triangle $T$, sweeping out the $2 J$ triangles $T_{j}$ having no common median vertex with $T$. The ovals in the triangles $T_{1}, T_{2}, \ldots, T_{2 J+2}$ appear successively in the cyclic ordering.

(4) If $\alpha=0$, then $\lambda_{+}-\lambda_{-}=0$

Proof The points (1), (2), (3) are easily proven using Bezout's theorem with conics. Notice that if $\alpha=0$, the pencils of lines $\mathcal{F}_{A_{i}}: A_{j} \rightarrow T_{j} \rightarrow A_{k}$ have no $\mathcal{J}$-jumps, and give rise to a closed Fiedler chain involving all of the empty ovals, hence (4) follows.

Definition 2 Let $C_{9}$ be an $M$-curve with deep nest, and no outer ovals. The curve $C_{9}$ has $J O_{1}$-jumps with distribution $\left(l_{1}, \ldots, l_{2 J+2}\right)$ if $C_{9}$ verifies 
the condition of Lemma 1 and $l_{i}, i=1, \ldots, 2 J+2$ are the cardinals of the successive groups of inner and median ovals in the cyclic ordering.

The case $J=1$ is illustrated in Figure 2. Notice that the number of $O_{1}$-jumps and their distribution is a rigid isotopy invariant of $C_{9}$.

Theorem 1 The real schemes $\langle\mathcal{J} \amalg 1\langle\beta \amalg 1\langle\gamma\rangle\rangle\rangle$, with $\beta+\gamma=26$ and $\beta$, $\gamma$ odd are not realizable by $M$-curves of degree 9 .

Among the 13 admissible schemes of that form, the 3 with $\beta=1,3$ and 25 had already been forbidden in [6].

Theorem 2 Let $C_{9}$ be an $M$-curve with real scheme $\langle\mathcal{J} \amalg 1\langle\beta \amalg 1\langle\gamma\rangle\rangle\rangle$, with even $\beta, \gamma$. Then:

1. if $C_{9}$ has $O_{1}$-jumps, then the complex scheme of $C_{9}$ is

$$
\begin{aligned}
& \left\langle\mathcal{J} \amalg 1_{-}\left\langle\left(\frac{\beta-6}{2}\right)_{+} \amalg\left(\frac{\beta+6}{2}\right)_{-} \amalg 1_{-}\left\langle\left(\frac{\gamma+6}{2}\right)_{+} \amalg\left(\frac{\gamma-6}{2}\right)_{-}\right\rangle\right\rangle\right\rangle \text {or } \\
& \left\langle\mathcal{J} \amalg 1_{+}\left\langle\left(\frac{\beta+4}{2}\right)_{+} \amalg\left(\frac{\beta-4}{2}\right)_{-} \amalg 1_{+}\left\langle\left(\frac{\gamma-4}{2}\right)_{+} \amalg\left(\frac{\gamma+4}{2}\right)_{-}\right\rangle\right\rangle\right\rangle .
\end{aligned}
$$

2. otherwise, the complex scheme of $C_{9}$ is

$$
\left\langle\mathcal{J} \amalg 1_{-}\left\langle\left(\frac{\beta+4}{2}\right)_{+} \amalg\left(\frac{\beta-4}{2}\right)_{-} \amalg 1_{-}\left\langle\left(\frac{\gamma}{2}\right)_{+} \amalg\left(\frac{\gamma}{2}\right)_{-}\right\rangle\right\rangle\right\rangle .
$$

Notice that by Theorem 2 , the real scheme $\langle\mathcal{J} \amalg 1\langle 2 \amalg 1\langle 24\rangle\rangle\rangle$ is not realizable. This real scheme was already forbidden in [6]. S. Orevkov constructed $M$-curves realizing the 10 real schemes $\langle\mathcal{J} \amalg 1\langle\beta \amalg 1\langle\gamma\rangle\rangle\rangle$, with $\beta$, $\gamma$ even, with $4 \leq \beta \leq 22$. Each of these curves has $3 O_{1}$-jumps, they realize the complex schemes $\left\langle\mathcal{J} \amalg 1_{+}\left\langle\left(\frac{\beta+4}{2}\right)_{+} \amalg\left(\frac{\beta-4}{2}\right)_{-} \amalg 1_{+}\left\langle\left(\frac{\gamma-4}{2}\right)_{+} \amalg\left(\frac{\gamma+4}{2}\right)_{-}\right\rangle\right\rangle\right\rangle$ (private communication).

\section{$2.2 \quad$ Lemmas}

Assume there exists an $M$-curve $C_{9}$ with real scheme $\langle\mathcal{J} \amalg 1\langle\beta \amalg 1\langle\gamma\rangle\rangle\rangle$.

We repeat hereafter the arguments from [6] for the case $\beta=0$ : let $A, B, C$ be 3 inner ovals. The pencils of lines $\mathcal{F}_{A}: B \rightarrow C, \mathcal{F}_{B}: C \rightarrow A$, and $\mathcal{F}_{C}: A \rightarrow B$ have no $\mathcal{J}$-jumps and give rise to a closed Fiedler chain involving all of the inner ovals. Therefore, $\lambda_{+}-\lambda_{-}=0, \Pi_{+}-\Pi_{-}= \pm 1$ and $\Lambda_{+}-\Lambda_{-} \in\{0,2,-2\}$. This contradicts the Rokhlin-Mishachev formula. The real scheme $\langle\mathcal{J} \amalg 1\langle 1\langle 26\rangle\rangle\rangle$ is not realizable.

Let now $\beta>0$. Assume $C_{9}$ has $O_{1}$-jumps. Let $\epsilon_{1}, \epsilon_{2}$ and $n \epsilon_{3}$ be respectively the contributions of $O_{1}, O_{2}$ and of the inner ovals to $\Lambda_{+}-\Lambda_{-}$(where 
$\epsilon_{1}, \epsilon_{2}, \epsilon_{3} \in\{+1,-1\}$, and $n$ is non-negative). By Lemma 1 (4) and the Rokhlin-Mishachev formula, one must have: $2\left(-\epsilon_{1} \epsilon_{2}-n \epsilon_{1} \epsilon_{3}\right)+\epsilon_{1}+\epsilon_{2}=8$. There are 4 possible solutions:

$$
\begin{aligned}
& \epsilon_{1}=\epsilon_{2}=-1, \epsilon_{3}=1, n=6 ; \\
& \epsilon_{1}=-1, \epsilon_{2}=\epsilon_{3}=1, n=3 ; \\
& \epsilon_{1}=1, \epsilon_{2}=\epsilon_{3}=-1, n=3 ; \\
& \epsilon_{1}=\epsilon_{2}=1, \epsilon_{3}=-1, n=4 .
\end{aligned}
$$

In the second case, one has $l_{+}=1, \pi_{-}^{+}-\pi_{+}^{+}=0$; in the third case, one has $l_{+}=1, \pi_{-}^{+}-\pi_{+}^{+}=3$. This contradicts the first Orevkov formula. Either of the other 2 cases verifies both Orevkov formulas. The numbers $n$, $\beta, \gamma$ have the same parity. If $C_{9}$ has $O_{1}$-jumps, these numbers are even, and Theorem 2 (1) is proven.

Lemma 2 Let $C_{9}$ be an $M$-curve with real scheme $\langle\mathcal{J} \amalg 1\langle\beta \amalg 1\langle\gamma\rangle\rangle\rangle$, without $O_{1}$-jumps and such that $\gamma \geq 2$ and $\beta \geq 1$. Let $A$ be a median oval, and $B, C$ be the extreme inner ovals met by the pencil $\mathcal{F}_{A}$ sweeping out $O_{1}$. The complete pencil $\mathcal{F}_{C}$ gives rise to a cyclic Fiedler chain involving all other empty ovals. In the corresponding cyclic ordering, all of the inner ovals are consecutive.

Proof: Let $T_{1}, \ldots, T_{4}$ be the 4 triangles $A B C, T_{1}$ and $T_{2}$ being the 2 ones with edge $[B C]$. The inner ovals lie in $Z_{1}^{\prime} \cup Z_{2}^{\prime}$, where $Z_{i}^{\prime}=T_{i} \cap \operatorname{Int}\left(O_{1}\right)$, $i=1,2$. One of the zones $Z_{i}^{\prime}, i=1,2$ is empty. Indeed, assume either zone contains an oval $E_{i}$. Then the conic through $A, B, C, E_{1}, E_{2}$ cuts $C_{9}$ in more than 18 points. Contradiction. Consider the pencil of lines $\mathcal{F}_{C}$ starting at $B$ and sweeping out the non-empty zone $Z_{i}^{\prime}, i \in\{1,2\}$. Let $D$ be the last inner oval met by this pencil. The pencil $\mathcal{F}_{C}: B \rightarrow D$ meets no median oval. Indeed, assume that a median oval $E$ is met by this pencil. Then, the conic $E B A D C$ cuts $C_{9}$ in more than 18 points. Contradiction. The complete pencil $\mathcal{F}_{C}$ gives rise to a cyclic Fiedler chain involving all other empty ovals. This chain splits into 2 consecutive subchains formed respectively by the inner and the median ovals.

Lemma 3 Let $C_{9}$ be an $M$-curve of degree 9 with deep nest, and 1 be any one of the inner ovals. Assume there exist five other empty ovals 2,3,4,5,6 met successively by the pencil of lines $\mathcal{F}_{1}^{+}$, and such that there is a $\mathcal{J}$-jump between any two successive ovals in the cyclic ordering. Denote by $\mathcal{F}_{1123456}$ the pencil of rational cubics through $1, \ldots, 6$ with double point at 1 . Then, up to cyclic permutation of $2, \ldots, 6$, the sequence of singular (i.e. reducible) cubics of $\mathcal{F}_{1123456}$ is: 
$16 \cup 14523,14 \cup 12356,12 \cup 14365,15 \cup 12643,13 \cup 15426$ or

$12 \cup 14365,15 \cup 12643,16 \cup 12543,13 \cup 12456,14 \cup 12356$ or

$16 \cup 15234,14 \cup 15326,15 \cup 13264,13 \cup 14265,12 \cup 14365$

See Figures 9, 10, 11.

Proof: Notice first that if $\mathcal{F}_{1}^{\epsilon}: X, Y, Z, X$ has a $\mathcal{J}$-jump between $X$ and $Y$ and between $Y$ and $Z$, then: $\mathcal{F}_{1}^{\epsilon}$ has also a $\mathcal{J}$-jump between $Z$ and $X$, and 1 lies in the principal triangle $X Y Z$. Thus, $1 \in 234 \cap 345 \cap 456 \cap 562 \cap 623$. As no three points among $1, \ldots, 6$ can be on a line, these five principal triangles must have a 2-dimensional intersection.

Case 1: The five points lie in convex position. Consider a pair of points, that are consecutive for $\mathcal{F}_{1}^{+}$, say 2,3 , and assume these points also consecutive for the convex cyclic ordering. This cyclic ordering is $2,3, X, Y, Z$, with $X, Y, Z \in\{4,5,6\}$. If $X, Y, Z=6,5,4$ or $5,6,4$, then $234 \cap 345=[34]$; if $X, Y, Z=6,4,5$ or $5,4,6$, then $632 \cap 456=6$; if $X, Y, Z=4,6,5$ or $4,5,6$, then $234 \cap 456=4$. Contradiction. Then, the only possible convex cyclic ordering of the five points is $2,4,6,3,5$. Choose a line at infinity $L$ that does not cut the convex hull of the points. In the oriented affine plane $\mathbb{R} P^{2} \backslash L$, there are a priori two possibilities for the positive cyclic convex ordering of the five points: $2,4,6,3,5$ and $2,5,3,6,4$. As $\mathcal{F}_{1}^{+}$sweeps out successively $2,3,4,5,6$, the first of these possibilities is realized. (see Figure 3).

Case 2: One of the points, say 2, lie in the convex hull of the other four. Assume 3 and 4 are consecutive in the cyclic convex ordering. Then, $234 \cap 562=2$. Contradiction. There are a priori two possibilities for the positive cyclic convex ordering of the four points: $3,5,4,6$ or $3,6,4,5$. In the first case, the quadrangle 3546 is divided into four triangles: $T_{1}=345 \cap 356$, $T_{2}=356 \cap 346, T_{3}=346 \cap 456$ and $T_{4}=345 \cap 456$. If $2 \in T_{1} \cup T_{2}$, then $623 \cap 456=6$; if $2 \in T_{3}$, then $623 \cap 345=3$. Contradiction. Hence, $2 \in T_{4}$. In the second case, the quadrangle 3645 is divided into four triangles: $T_{1}=346 \cap 356, T_{2}=356 \cap 345, T_{3}=345 \cap 456, T_{4}=346 \cap 456$. If $2 \in T_{1} \cup T_{4}$, then $632 \cap 345=3$; if $2 \in T_{2}$, then $623 \cap 456=6$. If $2 \in T_{3}$, then $1 \in 234 \cap 562$ and the pencil $\mathcal{F}_{1}^{+}$sweeps out successively $6,5,4,3,2$. This is a contradiction. Thus, $2 \in T_{2}$ (see Figure 4).

Case 3: Two of the points lie in the principal triangle determined by the other three. Assume the two points are not consecutive for $\mathcal{F}_{1}^{+}$, say these points are 4,6 . Then $234 \cap 345=[34]$. Contradiction. Thus, up to cyclic permutation of $2,3, \ldots, 6$, one can choose 4,5 as interior points. There are a priori two possibilities for the positive convex ordering of the other three points: $2,6,3$ and $2,3,6$. As $1 \in 632$ and $\mathcal{F}_{1}^{+}$sweeps out successively $6,2,3$, the latter possibility is excluded. The triangle 632 is divided in six 
triangles $T_{i}, i=1, \ldots, 6$ by the lines 42,43 and 46 , such that: $T_{1} \cup T_{2}=346$, $T_{3} \cup T_{4}=234, T_{5} \cup T_{6}=246$; and $T_{1}, T_{6}$ have 4,6 as common vertices, $T_{2}, T_{3}$ have 4,3 as common vertices, $T_{4}, T_{5}$ have 4,2 as common vertices. If $5 \in T_{1} \cup T_{2} \cup T_{4} \cup T_{5}$, then $345 \cap 456=[45]$; if $5 \in T_{6}$, then $456 \cap 562=[56]$. Contradiction. One has $5 \in T_{3}$ (see Figure 5).

Perform a Cremona transformation $\mathrm{cr}:\left(x_{0} ; x_{1} ; x_{2}\right) \rightarrow\left(x_{1} x_{2} ; x_{0} x_{2} ; x_{0} x_{1}\right)$ with base points 1,5,4. Let us denote the respective images of the lines $14,15,45$ by $5,4,1$. For the other points, we shall use the same notation as before $c r$. After $c r$, consider the pencil of conics $\mathcal{F}_{1236}$. This pencil has 3 singular conics: the double lines $12 \cup 36,13 \cup 26$ and $16 \cup 23$, they are shown in Figures 6, 7 and 8. The position of the pencil $\mathcal{F}_{1236}$ with respect to the base lines is uniquely determined, it is shown in the left-hand side of Figures 9,10 , and 11 . In cases 1,2 , this is obvious; in case 3 , both points 4,5 are swept out in the portion $16 \cup 23 \rightarrow 13 \cup 26$ and we have to find out which one is met first. The conic 16235 of $\mathcal{F}_{1236}$ is the image of a conic 15326 (see Figure 5), thus 4 lies outside of 16235 . So, 5 is swept out before 4 by $\mathcal{F}_{1236}$. Perform the Cremona transformation back. The pencil of conics $\mathcal{F}_{1236}$ is mapped onto the pencil of rational cubics $\mathcal{F}_{1123456}$ (right-hand side of Figures 9, 10, 11).

Lemma 4 Let $C_{9}$ be an $M$-curve of degree 9 with deep nest, and denote by 1 any one of the inner ovals. The complete pencil of lines $\mathcal{F}_{1}$ has at most 3 $\mathcal{J}$-jumps over the sequence of median ovals.

Proof Assume there exists an inner oval 1 of $C_{9}$ such that $\mathcal{F}_{1}$ has 5 $\mathcal{J}$-jumps over the median ovals. Let $2, \ldots, 6$ be median ovals such that $1,2, \ldots, 6$ verify the conditions of Lemma 3 . Notice that in the affine plane of Figures 9, 10, 11, a segment of cubic connecting two points among 1, .,6 goes over infinity if and only if it cuts $\mathcal{J}$. In each of the 3 cases, the cubics of the pencil $\mathcal{F}_{1123456}$ intersect all $O_{1} \cup O_{2} \cup\{1,2,3,4,5,6\} \cup \mathcal{J}$ at 27 points, so there is no possibility to sweep out the other empty ovals of $C_{9}$. Contradiction.

\subsection{Proof of Theorems 1 and 2}

Let $C_{9}$ be an $M$-curve of degree 9 with real scheme $\langle\mathcal{J} \amalg 1\langle\beta \amalg 1\langle\gamma\rangle\rangle\rangle$ and without $O_{1}$-jumps. Let $\epsilon_{1}, \epsilon_{2}$ and $n \epsilon_{3}$ be respectively the contributions of $O_{1}, O_{2}$ and of the median ovals to $\Lambda_{+}-\Lambda_{-}$; if $\gamma$ is odd, let $\epsilon_{4}$ be the contribution of the inner ovals to $\Lambda_{+}-\Lambda_{-}$(where $\epsilon_{1}, \epsilon_{2}, \epsilon_{3}, \epsilon_{4} \in\{+1,-1\}$ and $n$ is non-negative). Let $A$ be a median oval and $C$ be one of the extreme 


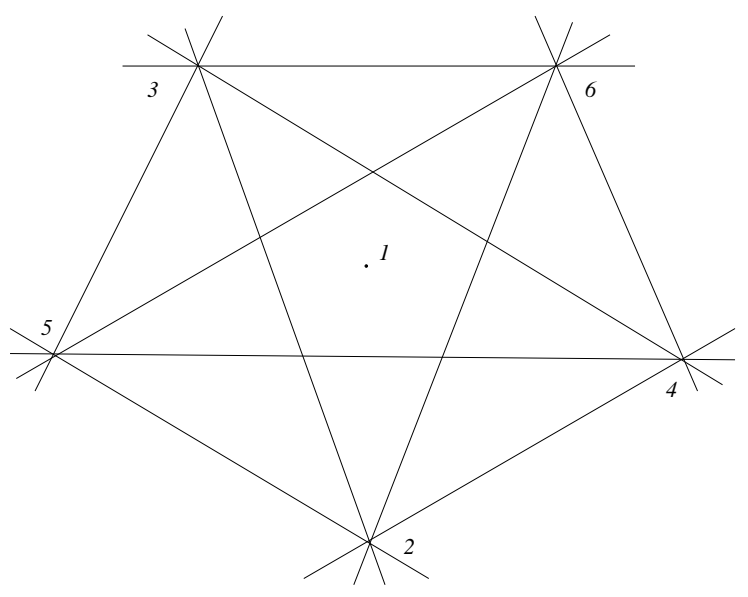

Figure 3: Case 1

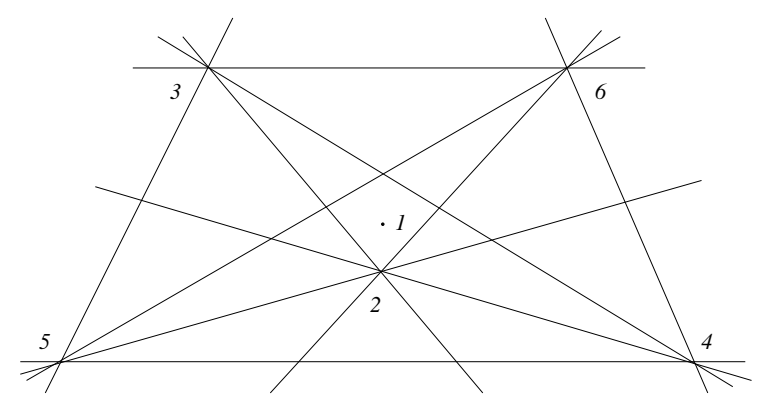

Figure 4: Case 2

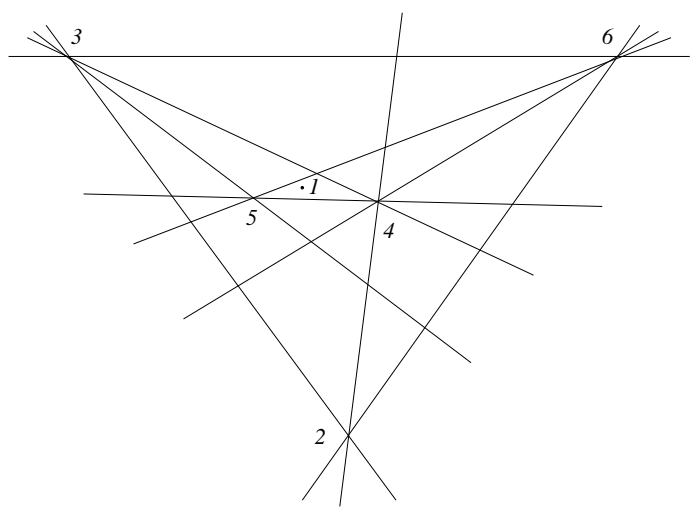

Figure 5: Case 3 


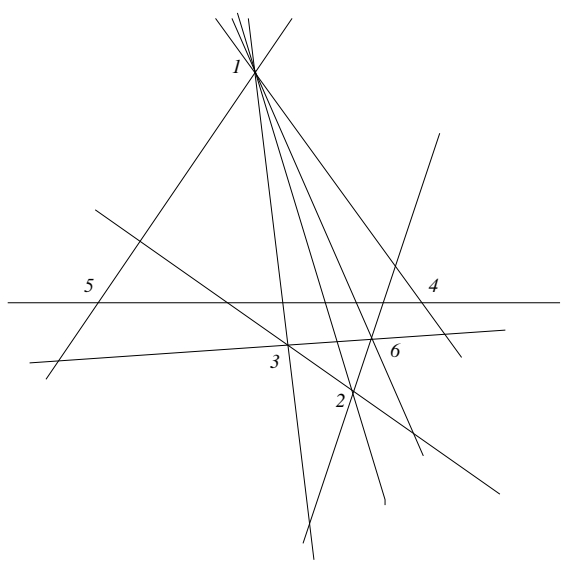

Figure 6 : The 3 double lines of $\mathcal{F}_{1236}$, case 1

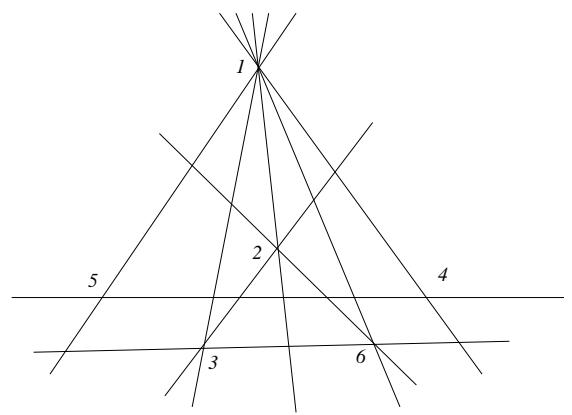

Figure 7: The 3 double lines of $\mathcal{F}_{1236}$, case 2

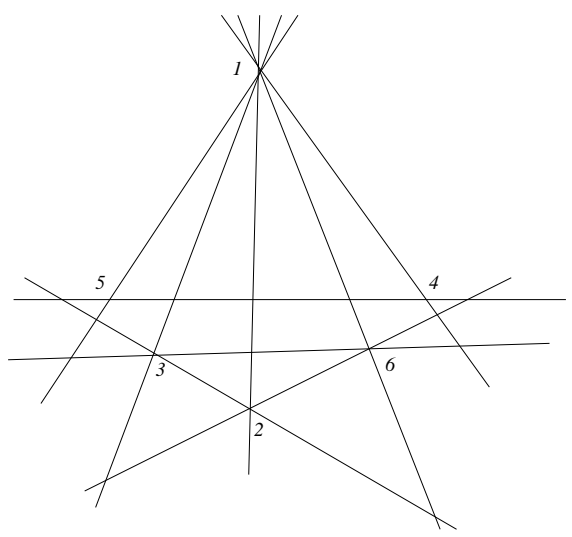

Figure 8: The 3 double lines of $\mathcal{F}_{1236}$, case 3 


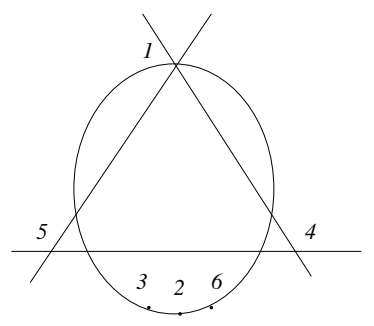

$16 \cup 23$
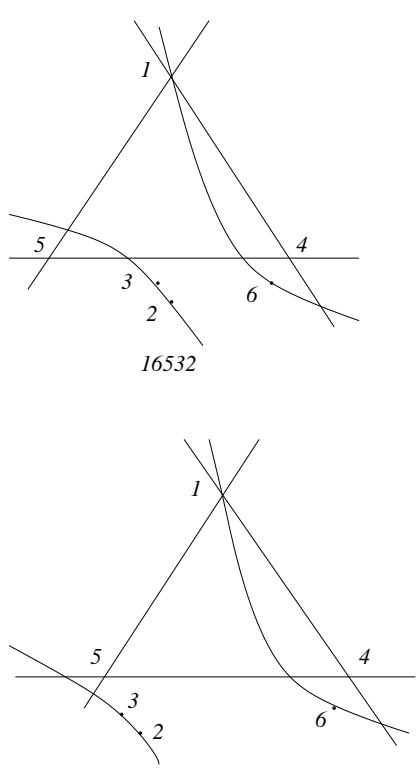

$12 \cup 36$
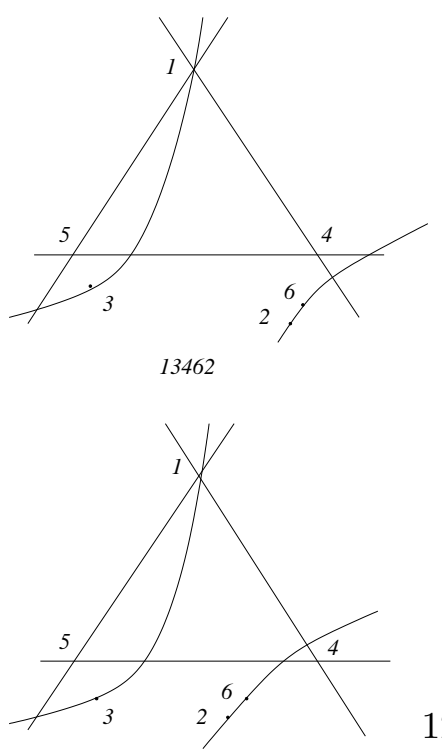

$13 \cup 26$
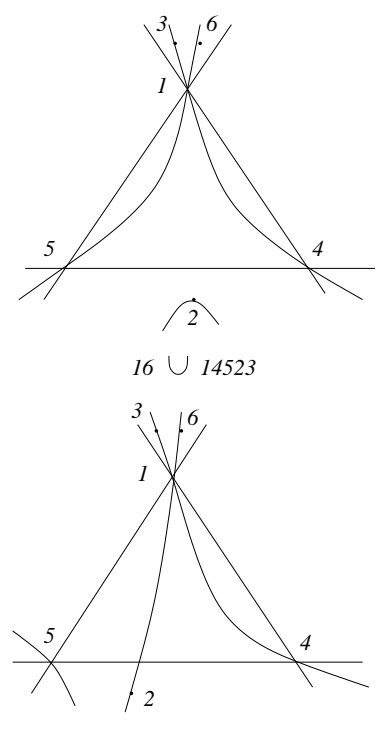

$14 \cup 12356$
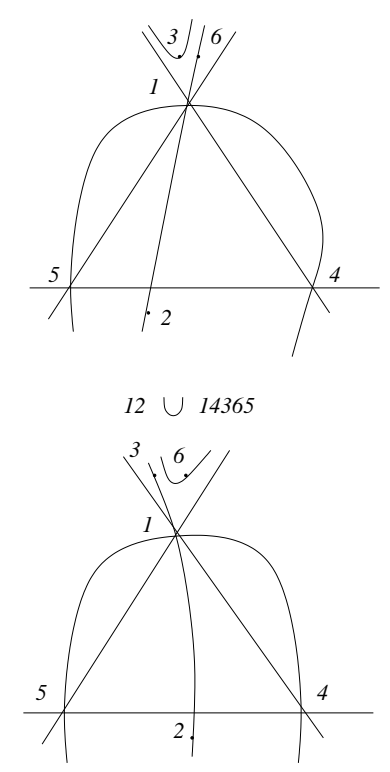

$15 \cup 12643$

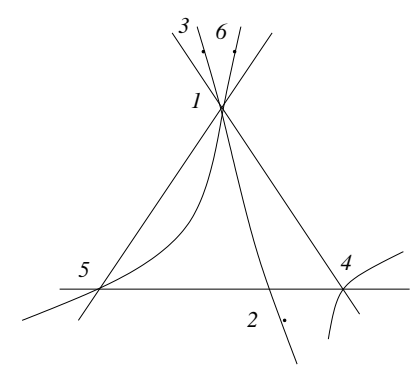

$13 \cup 15426$

Figure 9: $\mathcal{F}_{1123456}=c r^{-1}\left(\mathcal{F}_{1236}\right)$, case 1 

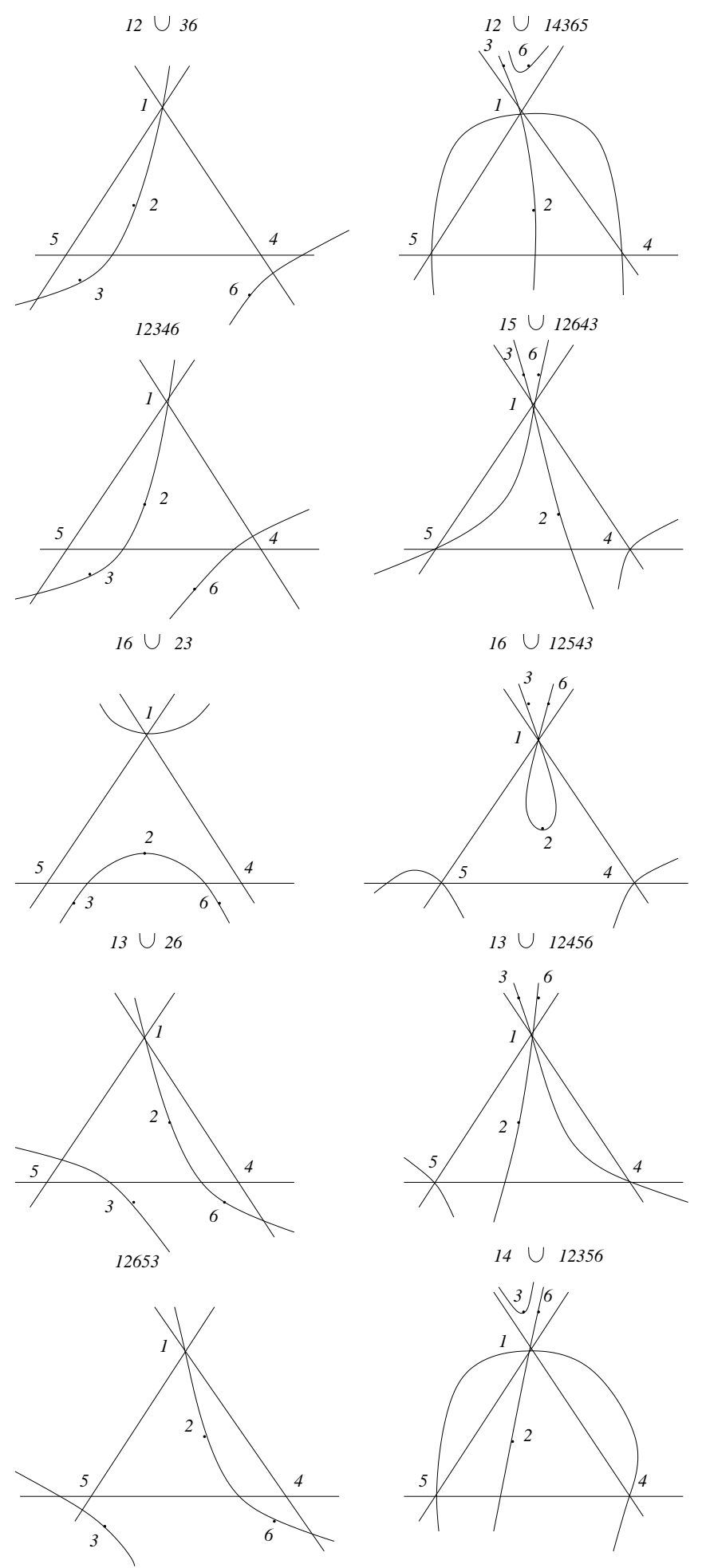

13

Figure 10: $\mathcal{F}_{1123456}=c r^{-1}\left(\mathcal{F}_{1236}\right)$, case 2 

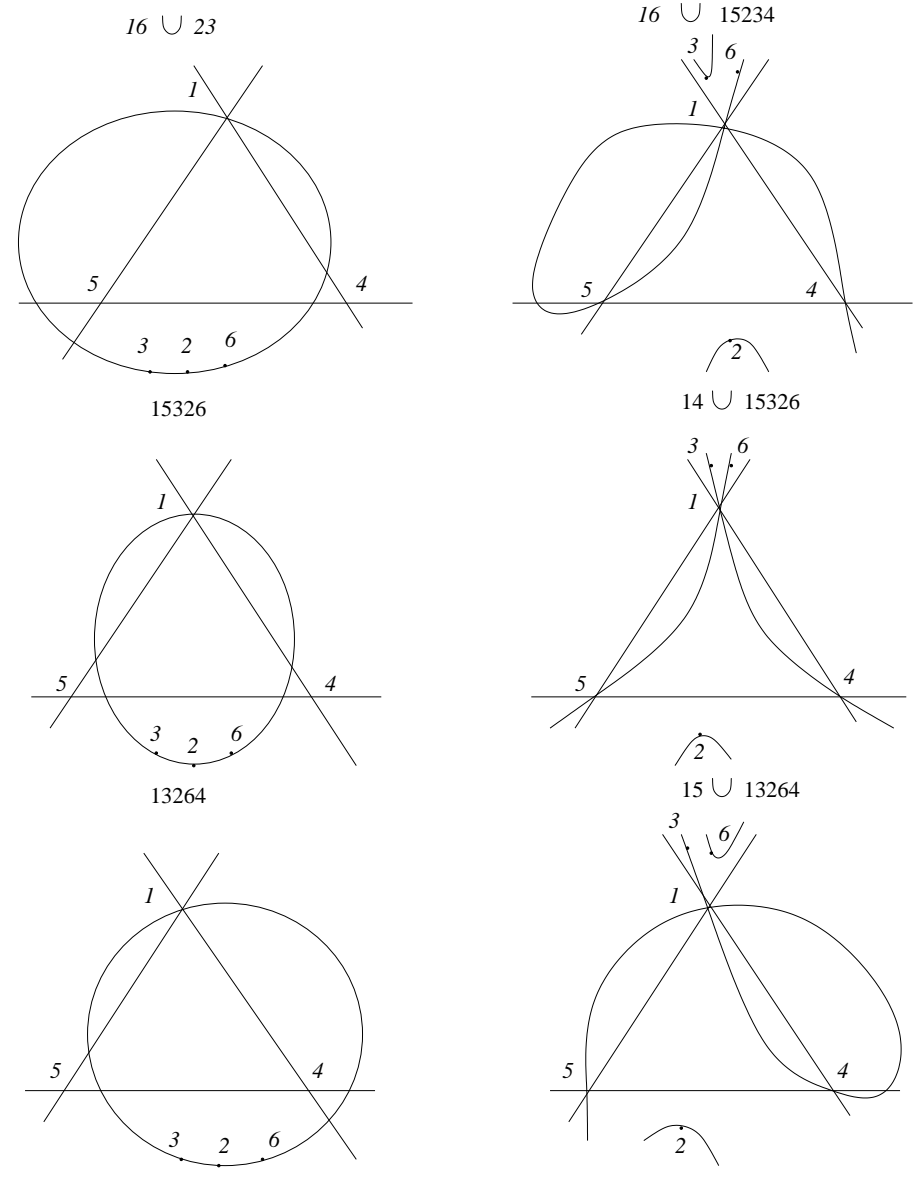

$13 \cup 26$
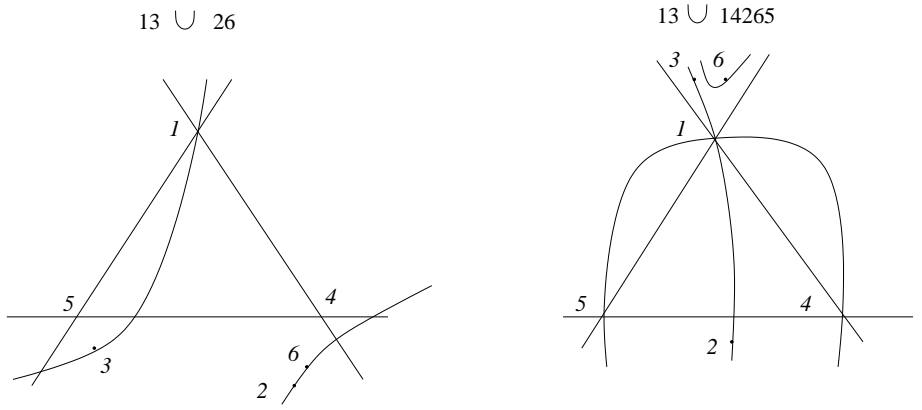

$12 \cup 36$
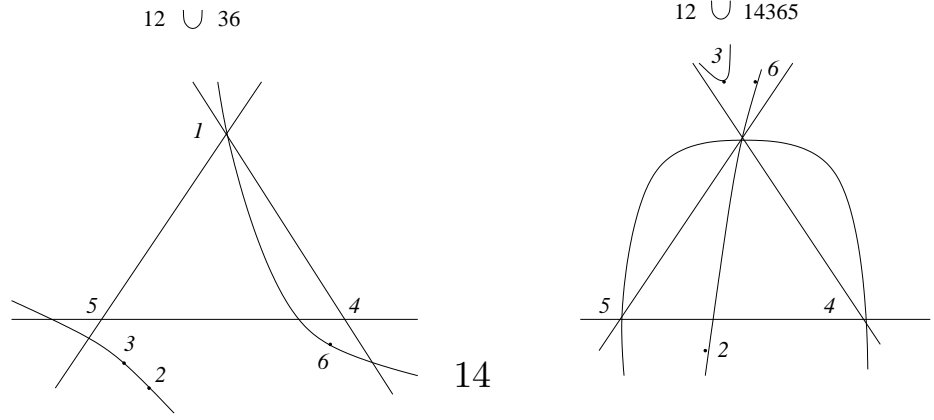

Figure 11: $\mathcal{F}_{1123456}=c r^{-1}\left(\mathcal{F}_{1236}\right)$, case 3 
inner ovals met by $\mathcal{F}_{A}$. Applying Lemmas 2 and 4 with the complete pencil of lines $\mathcal{F}_{C}$, we prove that if $\gamma$ is odd, then $n \in\{1,3\}$ and if $\gamma$ is even, $n \in\{0,2,4\}$. The Rokhlin-Mishachev formula yields respectively for odd and for even $\gamma$ :

$$
\begin{gathered}
2\left(-\epsilon_{1} \epsilon_{2}-\epsilon_{4} \epsilon_{2}-\epsilon_{4} \epsilon_{1}-n \epsilon_{3} \epsilon_{2}\right)+\epsilon_{1}+\epsilon_{2}+n \epsilon_{3}+\epsilon_{4}=8 \\
2\left(-\epsilon_{1} \epsilon_{2}-n \epsilon_{3} \epsilon_{2}\right)+\epsilon_{1}+\epsilon_{2}+n \epsilon_{3}=8
\end{gathered}
$$

For $\gamma$ odd, there is no solution. For $\gamma$ even, there are 2 solutions:

$\epsilon_{1}=\epsilon_{2}=-1, \epsilon_{3}=1, n=4$

$\epsilon_{1}=\epsilon_{3}=1, \epsilon_{2}=-1, n=2$.

In the latter case, one has: $l_{+}=1, \pi_{-}^{+}-\pi_{+}^{+}=0$. Contradiction with the first Orevkov formula. In the first case, both Orevkov formulas are verified. The numbers $n, \beta, \gamma$ have the same parity, if $C_{9}$ has no $O_{1}$-jump, these numbers are even. This finishes the proof of Theorem 1 and of Theorem 2 (2).

\section{References}

[1] T. Fiedler: Pencils of lines and the topology of real algebraic curves. Math. USSR Izvestia Vol.2 (1983) No 1.

[2] S. Fiedler-Le Touzé: Orientations complexes des courbes algébriques réelles. Thèse doctorale (January 2000).

[3] S. Fiedler-Le Touzé: Cubics as tools to study the topology of $M$-curves of degree 9 in $\mathbb{R} P^{2}$, J. London Math. Soc.(2) 66 (2002) 86-100.

[4] S. Fiedler-Le Touzé: Pencils of cubics as tools to solve an interpolation problem, Algebra and Application for Engeenering, Communication and Computing, special issue on Algebraic Curves, Vol.18 Number 1-2, February 2007, 53-70.

[5] A. Korchagin: Hilbert's 16th problem: history and main results. Visiting Schol. Lect. Math. Series, 19 (1997), Texas Tech. Univ., 85-140.

[6] A. Korchagin: M-curves of degree 9: new restrictions. Math. Notes, 39 (1986).

[7] A. Korchagin: Construction of new M-curves of 9th degree. Lect. Notes. Math., 1524 (1992), 296-307. 
[8] A. Korchagin: The new M-curves of degrees 8 and 9. Soviet. Math. Dokl., 39:3 (1989), 569-572.

[9] A. Korchagin: Smoothing of 6-fold singular points and constructions of 9th degree M-curves. Amer. Math. Soc. Transl. (1996) 173 (2), 141-155.

[10] S. Orevkov: Link theory and oval arrangements of real algebraic curves. Topology Vol.38 No 4. (1999) 779-810.

[11] S. Orevkov: Riemann existence theorem and construction of real algebraic curves. Annales de la Faculté des Sciences de Toulouse, Mathématiques (6) 12 (2003) no4, 517-531.

[12] S. Orevkov: Plane algebraic curves of odd degree with a deep nest J of Knot Theory and Its Ramifications, Vol 14, No. 4 (2005) 497-522

[13] S. Orevkov, O Viro: Congruence modulo 8 for real algebraic curves of degree 9. Russian Math. Surveys 56 (2001), 770-771.

[14] V. Rokhlin: Complex topological characteristics of real algebraic curves. Russian Math. Surveys, 33:5 (1978) 85-98.

[15] O. Viro: Progress in the topology of real algebraic varieties over the last six years. Russian Math. Surveys, 41:3 (1986) 55-82.

[16] O. Viro: Real algebraic plane curves: constructions with controlled topology. Leningrad Math. J. Vol.1 (1990) No 5.

[17] G. Wilson: Hilbert's sixteenth problem. Topology Vol.17 (1978) 53-73. 